\title{
Two-stage near-field source localization using acoustic vector sensor
}

\author{
Da-Wei Xiao, Jin-Fang Cheng and Chao-Ran Zhang \\ Dept. of Weaponry Engineering, Naval University of Engineering, Wuhan, 430033 China \\ Eamil:xdwmars@163.com
}

\begin{abstract}
To avoid 2-D search, a two-stage algorithm for passively estimating the azimuth and range of a near-field source using an acoustic vector sensor is proposed. This algorithm utilizes two particle velocity channels to estimate the azimuth, and then combines the pressure channel to estimate the range. The Cramér-Rao bound (CRB) on the estimation errors of the source azimuth is derived. Compared with the traditional method and CRB The proposed algorithm is computationally efficient and has the similar performance with 2-D MUSIC algorithm. Simulation results are provided to demonstrate the high-efficient quality of this method
\end{abstract}

Keywords: Near-Field; Localization; Acoustic Vector Sensor; Two-Stage.

\section{Introduction}

An acoustic vector sensor is different from the traditional acoustic sensor in which the output is a scalar corresponding to the acoustic pressure, a vector sensor measures a vector which consists of three orthogonally oriented acoustic particle velocities and the acoustic pressure, all co-located in space.

The passive localization of an acoustic source is of great importance in various fields. The traditional solution is usually done with sensor arrays, but as the vector sensor has complete acoustic information, this task can been done with a single sensor [1]. Two simple algorithms for estimation the source direction of arrival (DOA) with vector sensor and the mean-square angular error (MSAE) bound is introduced in [2]; the beamforming and Capon direction estimators have been applied to vector sensor in [3]; a maximum likelihood DOA estimator is derived in [4]; and the underdetermined DOA estimation using vector sensor has been recently appear in [5].

Nevertheless, the researches above all assume that the sources are in far-field, and the acoustic signal that received by the sensor is planar wave, but when the source move near to the sensor, the far-field assumption cannot be satisfied. A fourth-order cumulant based TLS-ESPRIT-like algorithm is proposed in [6], passive localization of mix near-field and far-field sources using 
two-stage MUSIC algorithm is appear in [7], and the near-field array-manifold of the acoustic vector-sensor is derived in [8-9]. However, most of these algorithms either require multidimensional search or high order cumulant, they need great amount of calculation. In this paper, we investigate a two-stage near-field source localization using an acoustic vector sensor. Firstly, we utilize two particle velocity channels to estimate the azimuth, and then combine the pressure channel to estimate the range. This method avoids the two-dimension grid search, and has the similar performance with 2-D MUSIC algorithm.

In section II, we state the mathematical model for the near-field measurement of a vector sensor. In section III, we consider the two-stage localization algorithm and derive the $\mathrm{CRB}$ of azimuth estimator. In section IV, we give some numerical examples and conclude the paper in $\mathrm{V}$.

\section{The Near-field Mathematical Model}

Throughout the paper, we assume that the wave is traveling in a quiescient, homogeneous, and isotropic fluid. Additionally, we assume the following:

A1) a simple signal will be used: The emitted signal is a pure tone with frequency $f$, an initial phase $\phi$ and complex-amplitude $P$.

A2) the snapshots are assumed to be statistically independent, the noise is complex Gaussian and spatiotemporally uncorrelated with zero mean and unknown deterministic covariances.

Under assumption A1, the spherical acoustic pressure signal can be expressed as

$$
p(\mathrm{r}, \mathrm{t})=\frac{P}{r} e^{j(\mathrm{wt}+\phi-\mathrm{kr})}
$$

Where $k$ denotes the wave number, according to acoustic Euler's equation [10], we can easy derive the near-field acoustic impedance

$$
Z(\mathrm{r}, \lambda)=\frac{p(r, t)}{v(r, t)}=\frac{\rho c}{1-j \frac{\lambda}{2 \pi r}}
$$

Where $\rho$ and $c$ denote, respectively, the medium density and acoustic velocity. In most case $\rho c$ is a constant. The near-field acoustic impedance is depend on range $r$ and wavelength $\lambda$, when $r \square \lambda$, the imaginary part of $Z(\mathrm{r}, \lambda)$ can be omit, the impedance will be as same as which in far-field situation.

The outputs of particle velocities of acoustic vector sensor is three orthogonally oriented components, FIG. 1 shows the geometry relation of each 
components, we have

$$
v_{x}=v(t) \cos \theta \cos \varphi, v_{y}=v(t) \sin \theta \cos \varphi, v_{z}=v(t) \sin \varphi
$$

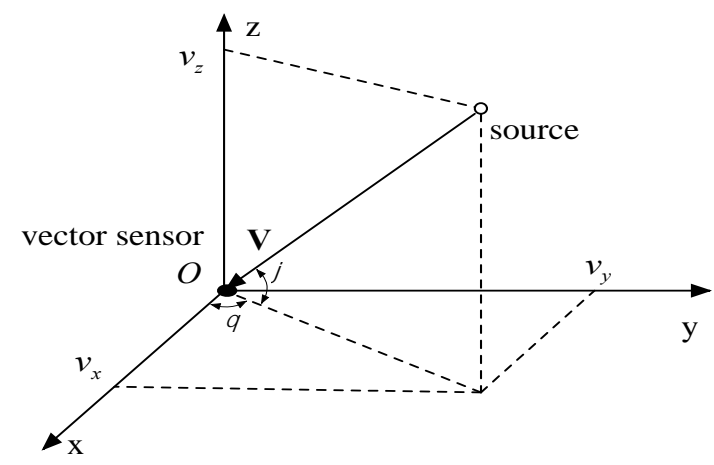

Fig. 1 Geometry relation of each particle velocities

Where $\theta$ and $\varphi$ denote, respectively, the azimuth and elevation angles of signal, combining (2) and (3), we have the outputs of acoustic vector sensor

$$
\left\{\begin{array}{c}
v_{x}=s(t) \cos \theta \cos \varphi \\
v_{y}=s(t) \sin \theta \cos \varphi \\
v_{z}=s(t) \sin \varphi \\
p=s(t) \frac{\rho c}{1-j \frac{\lambda}{2 \pi r}}
\end{array}\right.
$$

Consider the channel noise, the matrix expression of (4) is

$$
\mathbf{y}(t)=\left[v_{x}(t), v_{y}(t), v_{z}(t), p(t)\right]^{T}=\mathbf{a}(,, r) s(t)+\mathbf{n}(t)
$$

Where $\mathbf{a}(\theta, \varphi, r)$ is the near-field steering vector, $s(\mathrm{t})$ is the source signal. If only $\mathrm{x}-\mathrm{y}$ plane in the Cartesian coordinate system is considered (i.e., the elevation angle is 0$),(5)$ becomes

$$
\mathbf{y}_{x-y}(t)=\left[v_{x}(t), v_{y}(t), p(t)\right]^{T}=\mathbf{a}(\theta, r) s(t)+\mathbf{n}_{x-y}(t)
$$

Where $\mathbf{a}(\theta, r)=[\cos \theta, \sin \theta, \mathrm{Z}(\mathrm{r})]^{T}$ is a function of azimuth angle and range, usually we should estimate this two values simultaneously. Similar to the far-field MUSIC algorithm, the cost function of near-field 2-D MUSIC is

$$
\mathbf{F}(\theta, r)=\mathbf{a}^{H}(\theta, r) \mathbf{U}_{n} \mathbf{U}_{n}^{H} \mathbf{a}(\theta, r)
$$

Where $\mathbf{U}_{n}$ is the matrix of eigenvectors associated with the smallest eigenvalues of the data sample covariance matrix. Generally, the two-dimension 
grid search is needed to find the minimum of the cost function.

\section{Two-stage Localization}

In this section, a two-stage localization of near-field source is introduced using vector sensor, and the CRB of azimuth estimator is derived.

\subsection{Two-stage MUSIC algorithm}

The two-stage MUSIC algorithm uses two particle velocity channels only related with the azimuth and independent with the range, then combines this two velocity channels and pressure channel to estimate the range. This algorithm avoids the two-dimension grid search.

The two particle velocity channels signal can be expressed as

$$
\mathbf{F}(\theta, r)=\mathbf{a}^{H}(\theta, r) \mathbf{U}_{n} \mathbf{U}_{n}^{H} \mathbf{a}(\theta, r)
$$

Where the steering vector $\mathbf{a}()=[\cos , \sin ]^{T}$,
$\mathbf{n}_{v x-v y}(t)=\left[\mathbf{n}_{x}(t), \mathbf{n}_{y}(t)\right]^{T}$ denotes noise, when the sample time is $T$, the (8) becomes

$$
\mathbf{y}_{v x-v y}(m \Delta T)=\mathbf{a}(\theta) s(m \Delta T)+\mathbf{n}_{v x-v y}(m \Delta T)
$$

The data sample covariance matrix is

$$
\hat{\mathbf{R}}_{v x-v y}=\frac{1}{M} \sum_{m=1}^{M} \mathbf{y}_{v x-v y}(m \Delta T) \mathbf{y}_{v x-v y}^{H}(m \Delta T)
$$

The eigenvector associated with the small eigenvalue of $\hat{\mathbf{R}}_{v x-v y}$ is $\mathbf{u}_{n}$, so the cost function of MUSIC is

$$
\mathbf{F}_{1}(\theta)=\mathbf{a}^{H}(\theta) \mathbf{u}_{n} \mathbf{u}_{n}^{H} \mathbf{a}(\theta)
$$

We can estimate the azimuth $\theta$ by minimizing the cost function by one-dimension search, and then we take the estimated $\theta$ into (7), (7) become a function of range only

$$
\mathbf{F}_{2}(r)=\frac{1}{\mathbf{a}^{H}(r) \mathbf{U}_{n} \mathbf{U}_{n}^{H} \mathbf{a}(r)}
$$

So we can estimate the azimuth and range of near-field source.

\subsection{CRB of azimuth estimator}

To further characterize the estimator's performance, this subsection will derive the Cramér-Rao bound of the azimuth estimator. According to the assumption 
A1), the emitted signal is a pure tone with an single angular frequency and an initial phase; and the assumption A2), the snapshots are assumed to be statistically independent, the Fisher information matrix $\mathbf{J}$ with four deterministic unknown parameters $\chi=\left[\theta, \omega, \phi, \sigma_{v}^{2}\right]^{T}$ is [11]

$$
J_{i, j}=2 M \operatorname{Re}\left[\frac{\partial^{H} \boldsymbol{\mu}}{\partial(\chi)_{i}} \mathbf{R}^{-1} \frac{\partial \boldsymbol{\mu}}{\partial(\chi)_{j}}\right]+M \operatorname{tr}\left[\mathbf{R}^{-1} \frac{\partial \mathbf{R}}{\partial(\chi)_{i}} \mathbf{R}^{-1} \frac{\partial \mathbf{R}}{\partial(\chi)_{j}}\right]
$$

Where $\operatorname{Re}[\cdot]$ denotes the real-value part of the entity inside the square brackets, $\operatorname{tr}[\cdot]$ denotes the trace operation, and $(\boldsymbol{\chi})_{i}$ symbolizes the $i$ th element of $\boldsymbol{\chi}$. The fisher information matrix entries can be calculated with the statistical characteristics of received data, and $\mathbf{J}$ is block-diagonal

$$
\mathbf{J}=\left[\begin{array}{cccc}
J_{1,1} & 0 & 0 & 0 \\
0 & J_{2,2} & J_{2,3} & 0 \\
0 & J_{3,2} & J_{3,3} & 0 \\
0 & 0 & 0 & J_{4,4}
\end{array}\right]
$$

Hence, the CRB of the azimuth estimator is

$$
C R B(\theta)=J_{1,1}^{-1}=\frac{\delta_{v}^{2}}{2 M}
$$

\section{Numerical Example}

In this section, we present simulation results demonstrating the performance of the proposed algorithm, and compare to the 2-D MUSIC algorithm. The azimuth angle and range of the source is, respectively, $0^{\circ}$ and $3 \lambda$, the additive noise is assumed to be spatial white complex Gaussian, and the SNR is defined relative to each signal.

In the first example, the SNR is set to $10 \mathrm{~dB}$, and the number of snapshots is 400, FIG. 2 and FIG. 3 show the results of localization of two-stage MUSIC algorithm and 2-D MUSIC, respectively. We can see that both algorithms yield the location of the source successfully. 

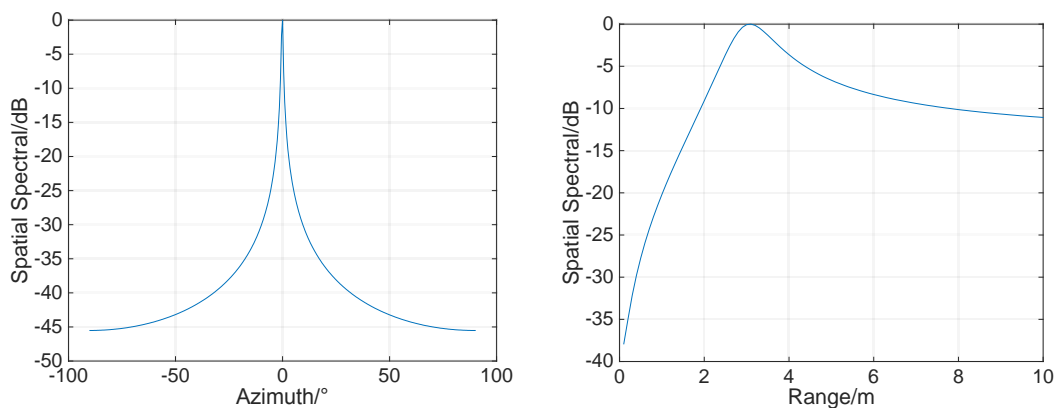

Fig. 2 The results of localization of two-stage MUSIC algorithm

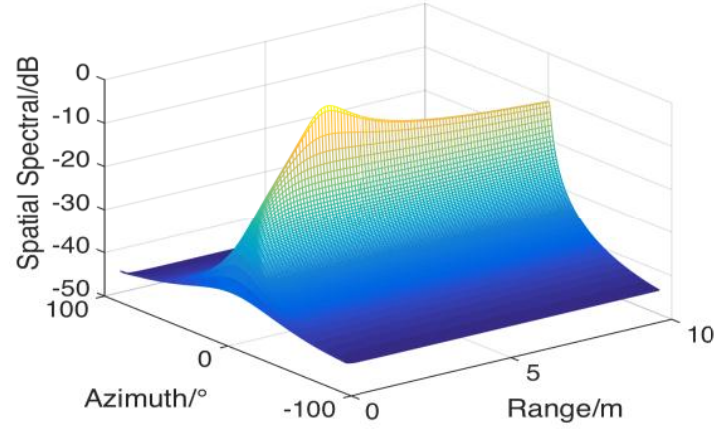

Fig. 3 The results of localization of 2-D MUSIC algorithm

Fig. 4(a) shows the second example, the number of snapshots is 400 , and the results are obtained from 400 independent Monte Carlo trials. The RMSEs and CRB of $\theta$ as a function of SNR are shown. From the figure, we can see that 2-D MUSIC algorithm has a better performance than two-stage algorithm at low SNR, but when the SNR $>10 \mathrm{~dB}$, two algorithm have the same performance, and close to the CRB.

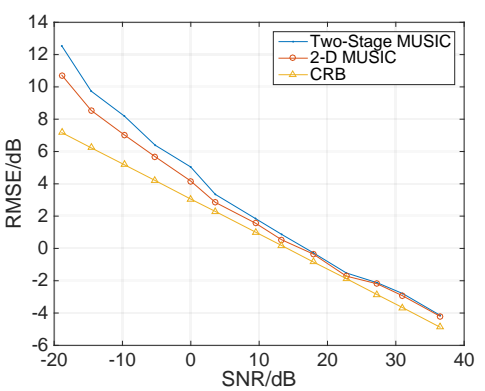

(a). RMSEs versus SNR

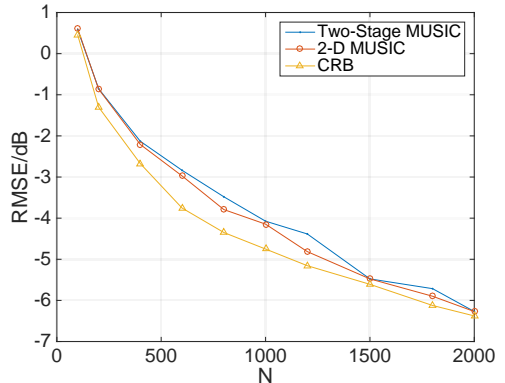

(b).RMSEs versus SNR

Fig. 4 RMSEs of azimuth estimates 
Fig. 4(b) shows RMSEs and CRB of $\theta$ as a function of the number of snapshots. The SNR is $15 \mathrm{~dB}$, and the results are obtained from 400 independent Monte Carlo trials. From the figure, we can see that 2-D MUSIC algorithm and two-stage algorithm have the same performance, and close to the CRB.

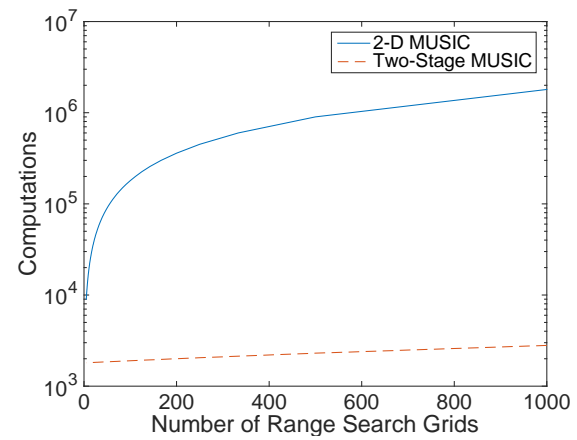

Fig. 5 Computational costs versus the number of range search grids

Fig. 5 shows the amount of calculation as a function of the number of angle search grids, it is seen from the figures that two-stage algorithm is computationally less complex than 2-D MUSIC algorithm.

\section{Conclusion}

Based on the property of the acoustic vector sensor, a two-stage algorithm is proposed to cope with the localization of a near-field source. The proposed method has moderate computation complex and has the similar performance with 2-D MUSIC algorithm. Simulation results are presented to verify the performance of this algorithm.

\section{Acknowledgment}

This work is partially supported by National Natural Science Foundation of China (50909096). The authors also gratefully acknowledge the helpful comments and suggestions of the reviewers, which have improved the presentation.

\section{References}

1. P. Tichavský, K. T. Wong and M. D. Zoltowski, Near-Field/Far-Field Azimuth and Elevation Angle Estimation Using a Single Vector Hydrophone, IEEE Trans. on Signal Processing, vol.49, no.11, pp.2498-2510, 2001. 
2. A. Nehorai and E. Paldi, Acoustic Vector-Sensor Array Processing, IEEE Trans. on Signal Processing, vol.42, no.9, pp.2481-2491, 1994.

3. M. Hawkes and A. Nehorai, Acoustic Vector-Sensor Beamforming and Capon Direction Estimation, IEEE Trans. on Signal Processing, vol.46, no.9, pp.2291-2304, 1998.

4. D. Levin, E. A. P. Habets and S. Gannot, Maximum likelihood estimation of direction of arrival using an acoustic vector-sensor, J. Acoust. Soc. Am., vol.131, no.2, pp.1240-1248, 2012.

5. S. Zhao, T. Saluev and D. L. Jones, Underdetermined direction of arrival estimation using acoustic vector sensor, Signal Processing, vol.100, pp.160-168, 2014.

6. R. N. Challa, and S. Shamsunder, High-Order Subspace-Based Algorithms for Passive Localization of Near-Field Source, Proc. of ASILOMAR-29, Pacific Grove, CA, pp.777-781, 1995.

7. J. L. Liang, and D. Liu, Passive Localization of Mixed Near-Field and Far-Field Sources Using Two-stage MUSIC Algorithm, IEEE Trans. on Signal Processing, vol.48, no.1, pp.108-120, 2010.

8. Y. I. Wu, K. T. Wong and S. Lau, The Acoustic Vector-Sensor's Near-Field Array-Manifold, IEEE Trans. on Signal Processing, vol.58, no.7, pp.3946-3951, 2010.

9. X. Yuan, Cramér-Rao Bounds of Direction-of-Arrival and Distance Estimation of a Near-Field Incident Source for an Acoustic Vector Sensor: Gaussian Source and Polynomial-Phase Source, IET Radar, Sonar and Navig., vol.6, iss.7, pp.638-648, 2012.

10. A. D. Pierce, A coustics-An Introduction to its Physical Principles and Applications, McGraw-Hill, New York, 1989.

11. H. L. V. Trees, Optimum Array Processing Part IV of Detection, Estimation, and Modulation Theory, John Wiley \& Sons, Inc, New York, 2002. 